\title{
Review of the Modeling of Wind Power Generation
}

\author{
Junru Shan \\ School of Electrical and Electronic Engineering, North China Electric Power University, Beijing \\ 102206, China \\ 1003434533@qq.com
}

Keywords: wind power, mathematical model, power system, power generation.

\begin{abstract}
Modeling of wind power plant is the basis for studying the influences that wind power has on electric power system. Based on the characteristics of wind power generation, this paper summarizes some common modeling methods, and puts forward the research prospects of wind power modeling.
\end{abstract}

\section{Introduction}

In recent years, with the deterioration of global environment and the shortage of energy, wind power generation, as the representative of new energy, has developed rapidly. It has also been widely concerned by scholars all over the world. It is foreseeable that the proportion of new energy power generation will gradually increase in the future so we must understand the impact when it is connected to the power grid. Due to the greater volatility and uncertainty, wind power generation is so different from the general thermal power plant and gas power plant. Only after we establish a new scientific mathematical model and have a comprehensive grasp of its operating mechanism, a reasonable scheduling is possible. Also, it will help us eliminate the harmonic pollution on the grid and improve the efficiency of the entire power system at the same time. In view of this problem, scholars have carried out a lot of research work and put forward a lot of relevant theoretical models. These models have their own advantages, disadvantages and the scope of use. To a certain extent, they have all been demonstrated by the practice. This paper mainly focus on the research of mainstream wind turbine and wind farm model. Finally, we have also put forward the future development of wind power.

Wind power generation can be divided into two types: off-grid and on-grid. Off-grid wind power generation is small and mainly used to solve the problem of electricity shortage in remote rural areas; on-grid wind power generation is the main way to use wind resources and under the compensation and support of the large-scale power system, it can make wind energy resources to be more fully developed. Also, it is the major research direction of wind power all over the world so in this paper we will mainly discuss it.

\section{Wind speed modeling}

The power of turbine is from wind energy, and wind speed is the major factor that affects wind power output. The wind speed model is independent from the wind turbine so it must be studied in order to make the characteristics of wind turbulence more accurately. Generally speaking, the existing theories are as follows: four-component model of wind, probability model, fuzzy model and interval model.

\subsection{The four-component model of wind}

After a lot of research and analysis, it is generally accepted that the natural wind consists of four components, namely, the basic wind, gust, gradient wind and random wind. They are explained as follows:

The basic wind: the stable component of wind which can maintain the same level of speed. Wind power station can record the changes of wind speed and draw it from the mathematical expectations of the Weibull distribution.

$$
V_{a}=A \times \Gamma\left(1+\frac{1}{K}\right)
$$


Where $\mathrm{A}$ is the scale parameter of the Weibull distribution, $\mathrm{K}$ is the shape parameter, and the algebraic $\Gamma\left(1+\frac{1}{K}\right)$ is the Gamma function.

Gust: This component describe the characteristic that wind speed may suddenly change. It is expressed as follows:

$$
V_{b}=\left\{\begin{array}{cc}
0 & \left(t<t_{q s}\right) \\
G \frac{1-\cos \left[2 \pi\left(t-t_{q s}\right)\right]}{t_{z z}-t_{q s}} & \left(t_{q s}<t<t_{z z}\right) \\
G\left(t-t_{z z}\right) & \left(t>t_{z z}\right)
\end{array}\right.
$$

Where $\mathrm{G}, t_{q s}$ and $t_{z z}$ represent the maximum wind speed, the start time and the end time of the gust, respectively.

Gradient wind: This component is to reflect the steady changes of wind energy. It represent the process that wind speed changes gradually with the passage of time. Gradient wind can be used to indicate that wind speed can change with the time gradient.

$$
V_{c}=\left\{\begin{array}{cc}
0 & \left(t<t_{k s}, t>t_{j s}+\Delta t\right) \\
C \frac{t-t_{k s}}{t_{j s}-t_{k s}} & \left(t_{k s}<t<t_{j s}\right) \\
C & \left(t_{j s}<t<t_{j s}+\Delta t\right)
\end{array}\right.
$$

Where $t_{k s}, t_{j s}, \Delta \mathrm{t}$ represent the start time, the end time, and the duration of the gradient wind, respectively. $\mathrm{C}$ is the maximum wind speed value obtained by the gradient wind.

(1) Random wind: Usually it can be expressed as the noise of wind. The expression is:

In this expression

$$
V_{d}=2 \sum_{i=1}^{N} \sqrt{S_{v}\left(\omega_{i}\right) \Delta \omega} \cos \left(\omega_{i}+\varphi_{i}\right)
$$

$$
\left\{\begin{array}{c}
\omega_{i}=\left(i-\frac{1}{2}\right) \Delta \omega \\
S_{v}\left(\omega_{i}\right)=\frac{2 K_{N} F^{2}\left|\omega_{i}\right|}{\pi^{2}\left[1+\left(\frac{F \omega_{i}}{\mu \pi}\right)^{2}\right]^{\frac{4}{3}}}
\end{array}\right.
$$

In the above two formulas, $\omega_{i}$ is frequency of each frequency segment, $\varphi_{i}$ is a random variable in the range of $0 \sim 2 \pi, K_{N}$ is the surface roughness coefficient, $\mathrm{F}$ is the random wind variation range, $\mu$ is the average wind speed at the relative height. $\mathrm{N}$ is the number of sample points collected.
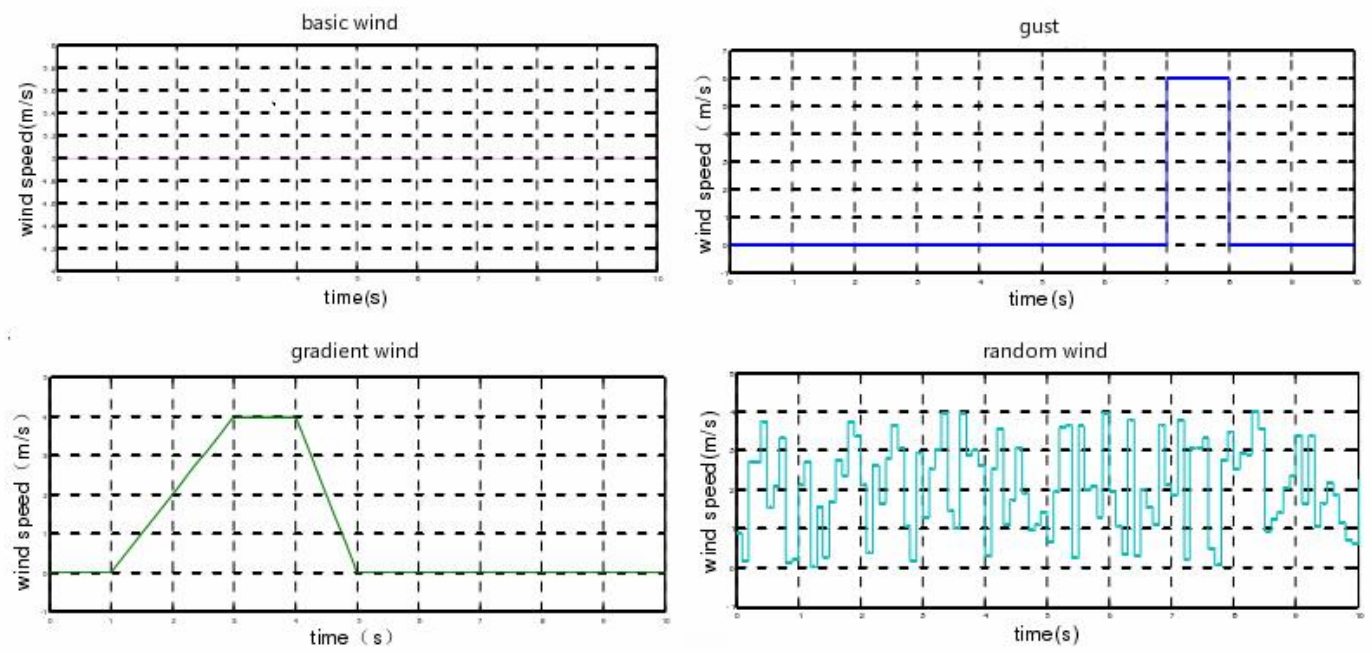

Fig.1 Four Different Component of wind 
Based on the four wind speed components proposed above, the equation for simulating the actual wind speed is as follows:

\subsection{Other models of wind speed}

$$
\mathrm{V}=V_{a}+V_{b}+V_{c}+V_{d}
$$

There are three other models to describe the uncertainty of wind speed: probability model, fuzzy model and interval model. The probability models of wind speed include Weibull distribution, Rayleigh distribution, Inverse Gaussian distribution and LogNormal distribution. Among them, the two-parameter Weibull distribution is one of the most widely used distributions. The two parameters (scale parameters and shape parameters) of the distribution can be calculated by analyzing the historical wind speed data. Common methods include maximum likelihood estimation, mean and variance estimation, least squares and so on. The fuzzy model of wind speed is based on the fuzzy mathematics theory, and the triangular fuzzy number and trapezoidal fuzzy number are used to describe the uncertainty of wind speed. It is mainly applicable to the case where the wind speed does not have statistical properties. The interval model of wind speed is based on the interval number theory, and using the interval number to characterize the uncertainty of wind speed is mainly used in situations where the wind speed prediction result is not accurate but accurate within a certain range.

\section{Mathematical model of wind turbine}

\subsection{Wind turbine and shaft model}

The energy transfer process of the wind turbine can be described as follows: the wind works to produce a pneumatic torque on the wind wheel to drive the shaft system. The shaft system then transmits the torque to the generator. In this process, the generator generates the reaction torque and acts on the wind wheel. Finally, the wind wheel speed reaches a balance value and complete the conversion of wind energy to mechanical energy.

Because of the complex mechanism of pneumatic, mechanical and other aspects, it is very difficult to obtain the accurate model of the wind turbine. Therefore, building a simplified wind turbine model to meet the research needs is the main method of wind turbine modeling.

Some scholars built the horizontal axis wind turbine aerodynamic performance model based on the slice theory. This model takes into account the influence of wind shear, yaw, structure parameters and installation parameters of the wind turbine. Also, we use the loss of the blade, the loss of the hub, the cascade theory and the failure of the momentum theory in the stall condition to amend this model. Another theory is to use the equivalent mass method to establish the equivalent model of wind turbine's three mass block which consider both the bending flexibility of the wind turbine and the drive shaft twist flexibility between the generator and the turbine. This model can be used for transient stability analysis; In addition, by using universal dynamic wake theory for wind turbine aerodynamics calculation and analysis, we can take into account the influence that structural deformation of wind turbine has on pneumatic performance and transmission performance. Using MATLAB/Simulink to carry out coupling analysis of vibration performance can also help set up corresponding models. Some scholars also pointed out that the input and output data of the experimental system can be used to build the multi-in and out stochastic model of the horizontal axis wind turbine system. By analyzing and testing it, we can find out the advantages and disadvantages in the design of the wind turbine control system. 


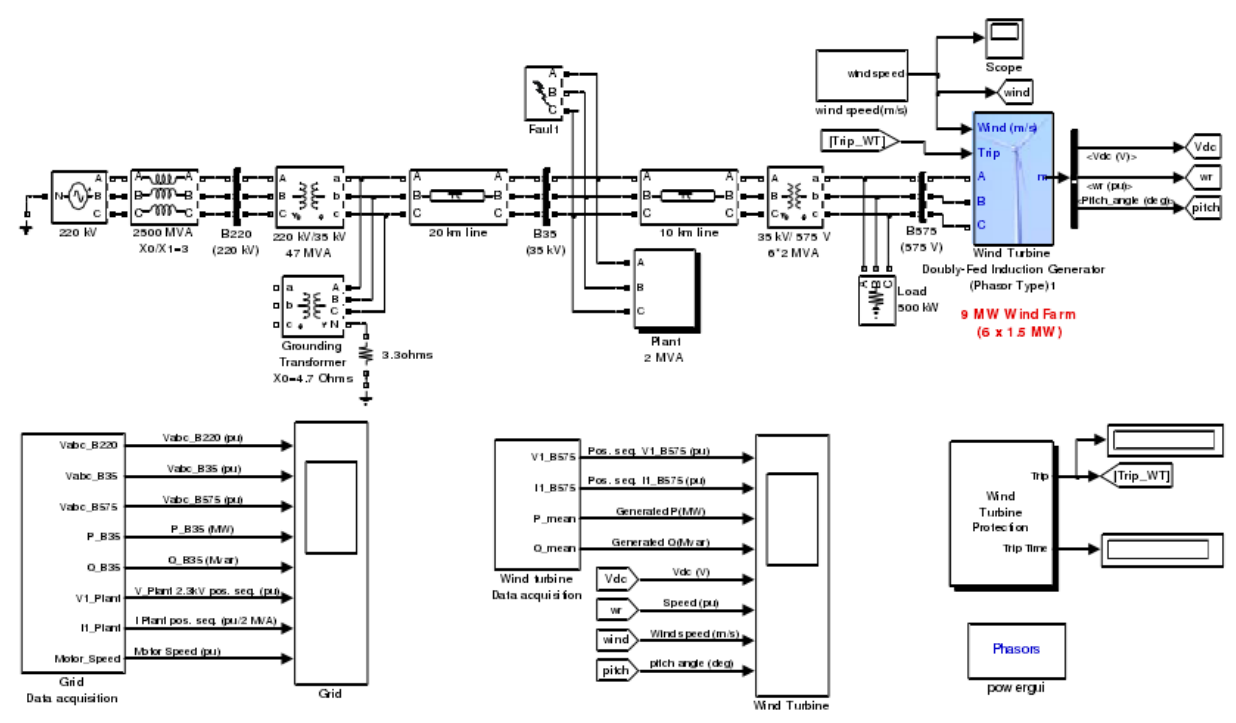

\subsection{Generator model}

Fig.1 A Wind Turbine Simulation System

At present, there are three types of wind turbine: constant speed wind turbine, doubly fed variable speed wind turbine and permanent magnet direct drive wind turbine. It involves different power generation control technologies such as constant speed constant frequency, variable speed constant frequency and variable speed frequency conversion. Universities such as the University of Aalborg in Denmark, the Danish Technical University, the University of Manchester, the Swedish Chalmers Technical University, and the University of Duisburg have conducted in-depth studies on wind turbine models. In addition, some countries have already developed a number of commercial power system analysis software, such as the PSCAD/EMTDC electromagnetic transient simulation program developed by the Manitoba High Voltage DC Research Center in Canada, the power system simulation program PSS/E developed by Siemens PTI Corporation and the PSLF power system analysis procedures developed by the United States General Motors.

\section{Wind farm model}

The wind farm model is usually used in the planning scheduling and optimization control of wind power system. It mainly describes the relationship between the output power of the wind farm and the wind speed. Due to the large number of wind turbines in the wind farm and the position effect of every unit, it's very difficult to establish an accurate wind farm model.

In general, the focus of various studies is the overall output characteristics of wind farms. Therefore, at present, the study of the output power of wind farms is largely simplified and the overall characteristics of wind farms are neglected. The existing research methods include: equivalent expansion of the output of different types of wind farm units; equivalent treatment of wind speed, and then calculate the output of the entire wind power.

\section{Discussion on the research direction of wind power modeling}

At present, the study of wind turbine characteristics and wind power modeling mostly aims at different kind of orphan problems so we haven't establish the essential link yet. Considering the reality that wind turbine itself is a more complex nonlinear system and contains a variety of variables and different time constant, it's a multi-time scale model system. So I believe that the modeling of wind power system should be able to reflect the impact of the following dynamic process. 


\subsection{The electromagnetic transient model determined by the dynamic process time constant of the converter}

Most modern large-capacity wind turbines have introduced flexible power electronic devices to improve the running performance, but the anti-interference ability of the power electronic devices is weak, and the wind turbines are mostly operated with constant power factor control. So when the grid voltage drops seriously, the turbine doesn't have the ability to withstand low voltage. In fact, when the grid voltage drops seriously (such as the occurrence of short circuit), in order to maximize the protection of turbine safety, the protection action will make the wind turbine off grid immediately. When the grid is large scale wind farm, this will intensify the degree of disturbance. To this end, in the study of this type of problem, we should pay more attention on that.

\subsection{Electromechanical transient model determined by the time constant of energy conversion process}

The fluid nature of the air determines the continuity of the wind speed, and the continuous change of the wind speed causes the wind power fluctuation to be continuous. When the wind speed is too large, it will lead the entire wind farm power shedding, which will cause a significant power impact to the power system. Especially, when the wind power is concentrated on a large-scale regional power system that use thermal power unit to adjust the frequency, the sudden changes of wind power may make the whole system exceed the response capacity. This is likely to cause the system frequency fluctuations and will even threaten the operation safe of the system.

We should consider the conversion process between the mechanical energy and electricity when we try to find how wind power will influence the grid. The wind power model should be able to reflect the electromechanical transient process under a certain time scale.

\subsection{Steady model of long-term dynamic process of wind energy fluctuation}

The output of wind power has strong random and large fluctuations so it's difficult to predict accurately. In order to study the influence of wind power on peak regulation and even grid planning, the model should be able to describe the fluctuation characteristics of the output power for a long time, such as the fluctuation range of wind power, fluctuation changes and other statistical indicators.

\section{References}

[1]. Andreas Petersson. Analysis, Modeling and Control of Doubly-Fed Induction Generators for Wind Turbines [D]. G',oteborg, Sweden: Chalmers University of Technology, 2005.

[2]. Francisco Jurado, Natividad Acero. USING VARIOUS COMPUTER TOOLS IN ELECTRICAL TRANSIENTS STUDIES [C] . 30th ASEE/IEEE Frontiers in Education Conference, Kansas City , MO ,October $18-21,2000$.

[3]. M. Poller. Doubly-Fed Induction Machine Models for Stability Assessment of Wind-Farms [C] . Proceedings of the IEEE Power-Tech Conference, Bologna, 2003.

[4]. J F Conroyand R Watson. Low-voltage ride-through Capability of Variable Speed Wind Turbines with Permanent Magnet Generators [J]. Wind Energ. Gener. ,2007, 1(3) :182-189.

[5]. Sophie Pelland. Solar and PV forecasting in Canada[C]. IESO Wind Power Standing Committee meeting, September 23, 2010, Toronto, Canada .

[6]. Eize de Vries. Where to next Developments and trends in wind turbines[J]. Renewable Energy World, 2012, 5(4): 62-71.

[7]. Morren J, Haan S W. Short-circuit current of wind turbines with doubly fed induction generator[J]. IEEE Transactions on Energy conversion, 2007,22(1):174-180 .

[8]. LuHao Lin. Research on Distributed Power Modeling and Control Strategy of Variable Speed Constant Frequency Wind Power System[D]. ChangSha University of Science and Technology, 2012, 27-33.

[9]. Xiong Liu, Yan Chen, ZhiQuan Ye. Pneumatic Performance Calculation Model of Horizontal Axis Wind Turbine [J]. Journal of Solar Energy. 2005, 26(6) :792-800 .

[10]. Hui Li, Li Han. Influence of Wind Turbine Equivalent Model on Unit Transient Stability Analysis[J] Journal of China Electromechanical Engineering, 2008, 28(17):105 - 111 . 
[11]. YaZhou Lei, Gordon Lightbody. Introduction of Forerign Wind Power Generation Guide and Dynamic Model[J].Power grid technology ,2005,29(12):27 -32 .

[12]. M. Lange, U.Focken. Approach to Short-Term Wind Power Prediction .Physical Berlin Springer 2006 .

[13]. T.Ackermann,L.Soder. An overview of wind energy-status 2002[J].Renewable and Sustainable Energy Reviews, 2002, 6 .

[14]. LIN Jikeng, LIU Lu, ZHANG Wenbo, at al. load modeling and parameters identification based on the random fuzzy syncretism analysis method[J]. Automation of Electric Power Systems, 2013, 37(14): 50-58 .

[15]. Li Xinran, HUI Jinhua, QIAN Jun, et al. Impact of wind power generation on load modeling in distribution network[J]. Automation of Electric Power Systems, 2009, 33(13): 89-94 .

[16]. ASMINE M, BROCHU J, FORTMANN J, et al. Model validation for wind turbine generator models[J]. IEEE Trans on Power Systems, 2011, 26(3): 1769-1782. 